\title{
A UK cost of care model for inflammatory bowel disease
}

\author{
Nivedita Ghosh, Purushothaman Premchand
}

Queen's Hospital, Barking, Havering \& Redbridge Hospitals NHS Trust, Romford, UK

\section{Correspondence to} Dr Nivedita Ghosh, Queen's Hospital, Barking, Havering \& Redbridge Hospitals NHS Trust, Rom Valley Way, Romford RM7 OAG, USA; nivedita_ghosh@hotmail.com

Received 25 October 2014 Revised 28 January 2015 Accepted 4 February 2015 Published Online First 24 February 2015

\section{CrossMark}

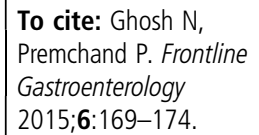

\begin{abstract}
Objectives There are an estimated 620000

patients with inflammatory bowel disease (IBD) in the UK. The rising incidence of IBD combined with its incurability has significant cost implications. The aim of this cost of care model was to calculate the annual cost per adult patient of treating ulcerative colitis (UC) and Crohn's disease (CD) from a NHS perspective, and to enable areas of potential cost savings to be explored.

Design The cost of IBD was calculated by summing the costs of treatment, treatment side effects and disease-related complications, accounting for the proportions of patients incurring these costs. Default input values for costs, the percentage of patients receiving each treatment, and the percentage of patients experiencing treatment-related side effects or disease-related complications were determined from national sources and published literature. However, the model permitted the user to input local or alternative data.
\end{abstract}

Setting The model was designed to be used by NHS trusts in the UK.

Results Using default input values, the annual cost of treating any patient with UC was estimated to be $\mathbf{f 3 0 8 4}$. For a patient with UC in remission, in relapse with mild-to-moderate UC or in relapse with severe UC, annual cost per patient was estimated to be $£ 1693, f 2903$ and $\mathrm{f} 10$ 760, respectively. The annual cost for any patient with CD was estimated to be $f 6156$ ( $£ 1800$ for patients in remission; $£ 10513$ for patients in relapse).

Conclusions While IBD remains a costly condition with modest potential cost savings, this model facilitates calculation of annual costs per patient with UC and CD, and its customisability will help hospitals identify areas where savings could be made.

\section{INTRODUCTION}

Inflammatory bowel disease (IBD) consists of the two main conditions, ulcerative colitis (UC) and Crohn's disease
(CD). UC is an inflammatory condition of the large bowel which starts in the rectum and can extend more proximally. It produces symptoms of diarrhoea and rectal bleeding. CD can affect any part of the gastrointestinal tract producing symptoms of diarrhoea, abdominal pain and weight loss. UC and CD are also associated with extragastrointestinal manifestations or complications such as skin rashes, arthritis and eye involvement among others. In about $5 \%$ of patients with IBD it is not possible to differentiate between features of UC and CD. These patients are categorised as having inflammatory bowel disease unclassified.

UC and CD are controllable but incurable diseases. They generally tend to become apparent in patients in their $20 \mathrm{~s}$ to $40 \mathrm{~s}$ and then display relapsing and remitting clinical characteristics. Currently the prevalence of IBD in the UK is $0.5-1 \%$ with an estimated 620000 people thought to be effected. ${ }^{1}$ The rising incidence of the condition, young age of onset and incurable nature mean that treatment of IBD has significant cost implications. The National IBD audit estimated the cost to the NHS in 2010 to be more than $£ 1$ billion with an average cost of $£ 3000$ per person per year. ${ }^{2}$ With the increasing cost of healthcare in general and the current economic climate financial considerations do need to be taken into account. The British Society of Gastroenterology has recently suggested a separately commissioned outpatient service for patients with IBD with commissioning according to a 'year of care' tariff. ${ }^{3}$

The cost of care for IBD includes the cost of treatment, the cost of treating or preventing adverse effects from treatment, and the cost of managing complications of the disease. Treatment options for UC include inexpensive topical therapy in the form of suppositories or enemas for distal colitis. For more 
extensive disease oral aminosalicylates are used for mild-to-moderate colitis, progressing to immunomodulators such as azathioprine or 6-mercaptopurine for disease not controlled on aminosalicylates. Steroids are used for flares. Hospitalisation and intravenous steroids may be required in patients with severe disease. With increased duration of UC there is an increased risk of bowel cancer. The risk of colorectal cancer at 20 years, 30 years and 40 years after the onset of disease is estimated at $3 \%, 8 \%$ and $11 \%$ and is reduced with surveillance colonoscopy. ${ }^{4}$ Of patients with UC $25 \%$ require surgery in the form of colectomy for either severe disease which does not respond to medical treatment or because of development of cancer or high grade dysplasia. ${ }^{5}$ With CD the mainstay of treatment is immunomodulatory drugs. Biological treatments such as anti-tumour necrosis factor (TNF) therapy are used for severe disease. CD is associated with complications such as abscesses, strictures and fistulae which have a significant impact on the patient's life. Of patients with CD $80 \%$ require surgery of some sort during their lifetime ranging from simple drainage of perianal abscesses to more complicated bowel resection. ${ }^{6}$

The cost of medication for IBD varies from relatively cheap aminosalicylates to more expensive biological therapies. Inpatient hospital care and surgery are also expensive. Biological treatment with anti-TNF therapy is costly; however, it reduces hospitalisation ${ }^{7}$ and need for surgery. ${ }^{8}$ The cost-effectiveness of these expensive treatments has now been recognised by the National Institute of Health and Care Excellence (NICE); NICE has recommended that the most severe $10 \%$ of patients with CD should receive anti-TNF therapy. There is also a cost associated with managing adverse effects from treatment such as pancreatitis from thiopurines or preventing osteopenia with steroids. Complications associated with the disease such as pyoderma gangrenosum or primary sclerosing cholangitis (PSC) carry a cost.

At present it is difficult for an individual gastroenterology unit to know how much they are spending annually per patient with IBD. We have developed a cost of care model which allows the annual cost of treating IBD per adult patient in an individual hospital to be determined. An important feature of the model is its customisability allowing users to input local data, thereby generating costs which are targeted and customised for that unit. This would be of benefit to individual gastroenterology units and commissioners alike. By providing a detailed breakdown of costs it allows identification of areas where savings may be made.

\section{METHODS}

\section{Model overview}

The cost of care model for IBD has been designed in a user-friendly Excel format. Two models have been developed, one for UC and one for $\mathrm{CD}$, in the same workbook. There is no separate model for inflammatory bowel disease unclassified but the cost of care for these patients can be incorporated within the UC or CD models. The cost of care for UC and CD is determined by the cost of treatment, cost of adverse effects resulting from treatment and the cost of complications, together with the proportion of patients incurring these costs. The model has been designed to calculate the average annual cost for a patient with UC and a patient with CD taking into consideration the proportion of patients in remission and in relapse, and the proportion of patients developing each of the major adverse effects of treatment or complications of the disease. For each input, the model provides a default setting which is determined primarily from national sources such as the British National Formulary, NICE, NHS trusts and from the published literature. However the model has been designed so that the user can input their own costs if known. The aim is that each individual unit will find out and enter its own costs thereby providing information that is customised and targeted for that unit. This can then be viewed and compared by local commissioning groups. The seven steps to customise the model are detailed below.

\section{Step 1-the cost of treatment}

The cost of care model first identifies the different possible treatment options with the associated costs. Medical and surgical, outpatient and inpatient treatment options are considered and include the various different mesalazine preparations available, other aminosalicylates, immunomodulators (azathioprine, 6-mercaptopurine, methotrexate), steroids, biological treatments (infliximab and adalimumab), leukapheresis, hospitalisation and surgery. Every major treatment option for UC and CD is included in the model. A sample of these can be found in table 1. Each treatment comes with a default cost calculated from national sources such as the British National Formulary (BNF)

Table 1 Costs of some of the major treatment options for UC and $C D^{9-12}$

\begin{tabular}{ll}
\hline Treatment & Cost per person per year \\
\hline Pentasa & $£ 517$ \\
Asacol & $£ 823$ \\
Azathioprine & $£ 95.88$ \\
6-mercaptopurine & $£ 540.96$ \\
Oral prednisolone & $£ 78.88$ \\
Infliximab & $£ 12584$ \\
Adalimumab & $£ 10368$ \\
Surgery for UC & $£ 16226.23$ \\
Hospital bed day & $£ 440$ \\
Outpatient consultation & $£ 128$ \\
\hline
\end{tabular}

$C D$, Crohn's disease; UC, ulcerative colitis. 
and NHS Trusts, with the option for local costs to be entered. $^{9-12}$ One benefit of this model is that as new treatment options become available the cost of these can be incorporated within the model. As patents for biological treatments expire and biosimilar copies become available the model has the option of including costs for the original drug and the biosimilar medication.

\section{Step 2-the cost of adverse effects}

The next step involves inputting the costs for adverse effects of treatment (see table 2). These include the costs of blood tests to monitor for possible side effects as well as the costs of treating adverse effects such as pancreatitis, myelotoxicity and hepatotoxicity arising from thiopurine use; osteopenia, osteoporosis and neuropsychiatric episodes from steroid treatment; infections as a result of immunosuppression; and complications of surgery. ${ }^{11} 12$ The cost of blood tests are included in the model but the cost of endoscopy or radiological imaging is not. The model provides national costs as a default but these can be customised to address local costs.

\section{Step 3-the cost of complications}

The third step addresses the cost of complications of diseases such as cancer, uveitis and iritis, pyoderma gangrenosum and PSC. The cost per person per year for surveillance for cancer is $£ 504$, for treating cancer $£ 10514$, the cost for uveitis and iritis $£ 125.66$, the cost for pyoderma gangrenosum $£ 463.04$ and the cost for PSC $£ 2099.25$. $^{9}$

\section{Step 4-the proportion of patients in remission and relapse}

The annual cost is dependent on the costs of treatment, side effects and complications, and on the proportion of patients on these treatments and developing side effects and complications (see tables $3-7)$. First the proportion of patients in remission and in relapse is entered. Remission is defined as in a clinical setting through a combination of clinical history, blood tests, imaging and radiology. Again national

Table 2 Costs of some of the adverse effects of treatment ${ }^{11} 12$

\begin{tabular}{|c|c|}
\hline Side effects/monitoring for side effects & $\begin{array}{l}\text { Cost per } \\
\text { person per year }\end{array}$ \\
\hline Blood tests & f152 \\
\hline Treating pancreatitis from thiopurine use & f2043.19 \\
\hline Treating myelotoxicity from thiopurine use & f2036.14 \\
\hline Treating hepatotoxicity from thiopurine use & $f 1209.81$ \\
\hline Osteopenia from steroids & f1134 \\
\hline Osteoporosis from steroids & $£ 2267$ \\
\hline Mild neuropsychiatric episode from steroid use & f1384 \\
\hline Severe neuropsychiatric episode from steroid use & $£ 2778$ \\
\hline Serious infections from biological treatment & £4095.65 \\
\hline Bleeding following balloon dilatation & f561.48 \\
\hline
\end{tabular}

Table 3 Some of the treatments for patients with UC in remission

\begin{tabular}{ll}
\hline Treatment & Percentage of patients \\
\hline Oral mesalazine & 65 \\
Oral sulphasalazine & 17 \\
Azathioprine & 3 \\
6-mercaptopurine & 2 \\
\hline A patient may be on more than one treatment. & \\
UC, ulcerative colitis. &
\end{tabular}

estimates come as default but local proportions can be entered. It is estimated that $50 \%$ of patients with UC are in remission and 50\% in relapse. ${ }^{13}$ Of those in relapse $80 \%$ have mild-to-moderate disease and $20 \%$ severe disease.

\section{Steps 5, 6 and 7}

Then the proportion of patients on the various treatments are entered (step 5), followed by the proportion of patients developing side effects of treatment (step 6) or complications of disease (step 7).

Using the cost of treatment, the cost of side effects, the cost of complications and the proportions of patients in remission and relapse, on each type of treatment, developing side effects and complications, the annual cost per head is then calculated for UC and CD.

In CD 50\% of patients are in remission and $50 \%$ in relapse. Some relapsing patients require hospitalisation with an average stay of 7 days. Of patients that are admitted to hospital $7.4 \%$ are given anti-TNF treatment and $18 \%$ require surgery. ${ }^{2}$

\section{RESULTS}

Using the default cost settings provided by the model, for UC the cost per patient in remission is $£ 1693.25$ per year, in mild-to-moderate relapse is $£ 2903.24$ per year, and with severe disease is $£ 10760.23$ per year. Overall, this works out to an average of $£ 3083.94$ per patient with UC per year (see table 8).

In $C D$ the cost per patient in remission is $£ 1799.85$ per year. The cost per patient in relapse is $£ 10$ 513.03. The average annual cost for any patient with CD is $£ 6156.44$ (see table 9).

Table 4 Some of the treatments for patients with UC in mild-to-moderate relapse

\begin{tabular}{lc}
\hline Treatment & Percentage of patients \\
\hline Topical aminosalicylates & 30 \\
Oral mesalazine & 100 \\
Topical corticosteroids & 30 \\
Oral prednisolone & 50 \\
Oral tacrolimus & 0.5 \\
Leukapheresis & 0.5 \\
\hline A patient may be on more than one treatment. & \\
UC, ulcerative colitis. &
\end{tabular}


Table 5 Some of the treatments for patients with UC with severe disease

\begin{tabular}{lc}
\hline Treatment & Percentage of patients \\
\hline Hospitalisation & 100 \\
Intravenous hydrocortisone & 100 \\
Ciclosporin & 10 \\
Infliximab & 7 \\
Surgery & 20
\end{tabular}

A patient may be on more than one treatment.

UC, ulcerative colitis.

Using the model, there is then the opportunity to see how costs could be reduced. This could be done by changing to a cheaper drug within the same class (eg, a cheaper 5-aminosalicylate (ASA) or anti-TNF) or by increasing the use of a more costly drug but thereby increasing the proportion of patients in remission or reducing the need for hospitalisation or surgery.

For example, the costs of mesalazine by brand are: Salofalk, $£ 341$ per person per year; Pentasa, $£ 517$ per person per year; Mezavant, $£ 874$; Asacol, $£ 823$; and Octasa, $£ 664 .{ }^{9}$ Of the patients with UC on mesalazine the default proportion using each brand are: Salofalk, 0\%; Pentasa, 25\%; Mezavant, 10\%; Asacol, 60\%; Octasa, $5 \%$, with an average annual cost of $£ 743.65$. Changing the proportion of patients receiving Asacol to $5 \%$ and the proportion of patients receiving Octasa to $60 \%$ would change the annual cost of mesalazine to $£ 656.20$ per year. It is however important to remember that the different brands are made up of different preparations of mesalazine with different modes of drug release and therefore may have different effects on a patient.

Hospitals can also calculate the costs if the percentage of patients on biologics is increased. If the percentage of patients with $\mathrm{CD}$ in relapse on adalimumab increases from $5 \%$ to $10 \%$ the cost rises from $£ 10513$ to $£ 11032$. The overall annual cost for any patient with CD increases from $£ 6156$ to $£ 6416$. Studies have shown that patients on specialty drugs

Table 6 Some of the adverse effects from thiopurines and infliximab $^{11} 12$

\begin{tabular}{ll}
\hline Side effect & Percentage of patients \\
\hline Allergic reactions from thiopurines & 2 \\
Pancreatitis resulting from thiopurines & 3.3 \\
Severe myelotoxicity from thiopurines & 2 \\
Hepatotoxicity from thiopurines & 0.3 \\
Blood tests for patients on thiopurines & 100 \\
Serious infection from infliximab & 0.0604 \\
Lymphoma in patients on infliximab & 0.001 \\
Demyelination & 0.0061 \\
Anaphylaxis & 0.5 \\
\hline
\end{tabular}

Table 7 Some of the complications of $U^{14-17}$

\begin{tabular}{ll}
\hline Complication & Percentage of patients \\
\hline Colorectal cancer & 3.7 \\
Surveillance for cancer & 0.03 \\
Uveitis/iritis & 5 \\
Pyoderma gangrenosum & 1.2 \\
PSC & 5.0 \\
\hline
\end{tabular}

PSC, primary sclerosing cholangitis; UC, ulcerative colitis.

such as biologics account for a large proportion of the annual total cost of care. ${ }^{18}$

The effect of increasing the use of leukapheresis can also be investigated. If the proportion of patients with mild-to-moderate colitis receiving leukapheresis were increased from $0.5 \%$ to $8 \%$ the cost in this group would increase from $£ 2903$ per year to $£ 3352$. The cost for any patient with UC would increase from $£ 3083$ to $£ 3263$. However if this results in a decrease in the annual proportion of patients developing acute severe colitis from $20 \%$ to $15 \%$, although the cost per person in the mild-to-moderate group remains at $£ 3352$ per year, the cost for any patient with UC becomes $£ 3078$.

\section{DISCUSSION}

\section{Validation of the model}

The annual cost of care for UC and CD calculated using the cost of care model is similar to the costs found in other studies. Bassi et $a l^{19}$ found the mean cost for UC to be $£ 1256$ and CD to be $£ 1652$, for 6 months. However, this study from 2004 took place at a time when there was limited use of biological therapy and so costs can be presumed to have risen since then. The national IBD audit estimated the cost of IBD to be $£ 3000$ per person per year but did not include the cost of treating complications. ${ }^{2}$

\section{Limitations and strengths of the model}

A key limitation of this model is that the effectiveness and utility of treatment are not evaluated. Ultimately physicians should and are required to make decisions for treatment on a patient-by-patient basis.

Additionally, the model inputs are based upon a number of assumptions. For example, treatment costs for the oral mesalazines were calculated on the dosing frequency and duration indicated by the BNF guidance. In general, model inputs were derived from national figures. However, a few model inputs, such as the cost of complications due to azathioprine were derived from a Spanish study by Panés et al. ${ }^{11}$ These costs were converted from Euros to GBP using the historic exchange rate for 2004 and were upscaled to GBP 2013 using consumer price index (CPI) average inflation rate by year.

A key strength of this model is its customisability. This allows information to be generated which is specific to a local hospital and its community and is therefore more accurate. 
Table 8 Cost per patient with UC per year

\begin{tabular}{lccrr}
\hline & Treatment & Adverse effects & \multicolumn{1}{c}{ Complications } & Total cost \\
\hline Clinical remission & $£ 891.18$ & $£ 40.60$ & $£ 761.47$ & $£ 1693.25$ \\
Relapse with mild-to-moderate disease & $£ 1263.57$ & $£ 878.19$ & $£ 761.47$ & $£ 2903.24$ \\
Relapse with acute severe disease & $£ 8016.82$ & $£ 1981.94$ & $£ 761.47$ & $£ 10760.23$ \\
Any patient with UC & $£ 1752.70$ & $£ 569.77$ & $£ 761.47$ & $£ 3083.94$ \\
\hline
\end{tabular}

UC, ulcerative colitis.

\section{Identifying areas of cost reduction}

The model allows areas of cost savings to be identified. For example a gastroenterology unit could explore the potential cost savings if they were to change from one brand of mesalazine to another. Therefore, using this model, budget holders are able to quantify the expected impact of healthcare decisions on hospital expenditure.

Importantly, the model accounts for the treatment cost, and the cost of adverse events occurring due to each treatment. This is a particularly important consideration in the case of biological and surgical therapies, which carry substantial costs due to complications of treatment.

The model also allows exploration of cost savings if leukapheresis were to be used more frequently than it currently is in the UK. Increasing the percentage of patients with mild-to-moderate UC on leukapheresis from $0.5 \%$ to $8 \%$ increased the annual cost per mild-to-moderate patient from $£ 2903$ to $£ 3352$, and the annual cost for any patient with UC from $£ 3083$ to $£ 3263$. However, assuming that increased use of leukapheresis would cause a decrease from 20\% to $15 \%$ in the annual proportion of patients experiencing acute severe flares, the annual cost for any patient with UC fell to $£ 3078$.

\section{CONCLUSION}

This is a detailed but simple to use cost model for IBD which allows calculation of annual cost per head for UC and CD for individual gastroenterology units and commissioners. It also allows areas for possible savings to be investigated. IBD will still remain a costly condition and any savings will be modest but the model can help with local cost breakdown and possible areas for savings.

Table 9 Cost per patient with CD per year

\begin{tabular}{|c|c|c|c|c|}
\hline & Treatment & $\begin{array}{l}\text { Adverse } \\
\text { effects }\end{array}$ & Complications & Total \\
\hline Clinical remission & f975.59 & f145.83 & f678.43 & f1799.85 \\
\hline Clinical relapse & $£ 8627.50$ & f1207.10 & f678.43 & f10 513.03 \\
\hline $\begin{array}{l}\text { Any patient with } \\
C D\end{array}$ & f4801.54 & f676.43 & f678.43 & f6156.44 \\
\hline
\end{tabular}

CD, Crohn's disease.

\section{Significance of this study}

\section{What is already known}

- As a chronic incurable condition the management of inflammatory bowel disease (IBD) has significant cost implications.

- There are publications which have looked at either the annual cost of treating IBD or looking at the costeffectiveness of treatments.

- However to our knowledge there is no cost of care model in the UK setting which accounts for the cost of treatments, side effects and complications.

\section{What this paper adds}

- This cost of care model allows annual average costs per patient with ulcerative colitis (UC) and Crohn's disease (CD) to be calculated by adding together the breakdowns of cost of treatment, cost of side effects and cost of complications.

- By allowing individual hospitals to input their own costs it produces values which are targeted and customised for each hospital.

How might it impact on future clinical practice

- The model could be used by individual hospitals to calculate their average annual cost of care for adult patients with UC and CD.

> Potential areas of cost savings could be explored.

- By allowing comparison in costs between different hospitals it could be used by NHS commissioning groups to determine where a patient with IBD could receive treatment.

Acknowledgements The authors thank Costello Medical Consulting, Cambridge, UK, for their support in developing the Cost of Care Model for IBD.

Competing interests None.

Provenance and peer review Not commissioned; externally peer reviewed.

\section{REFERENCES}

1 Molodecky NA, Soon IS, Rabi DM, et al. Increasing incidence and prevalence of the Inflammatory Bowel Diseases with time, based on systematic review. Gastroenterology 2012;142:46-54.

2 Report of the results for the national clinical audit of adult inflammatory bowel disease inpatient care in the UK. UK IBD Audit. London: RCP, 2012. 
3 Commissioning evidence-based care for patients with gastrointestinal and liver disease. BSG. http://www.bsg.org.uk/ images/Commissioning_report/BSG_Commissioning\% 20Report.pdf

4 Rutter M. Thirty-year analysis of a colonoscopic surveillance program for neoplasia in Ulcerative Colitis. Gastroenterology 2006;130:130-8.

5 Jess T, Riis L, Vind I, et al. Changes in clinical characteristics, course, and prognosis of inflammatory bowel disease during the last 5 decades: a population-based study from Copenhagen, Denmark. Inflamm Bowel Dis 2007;13:481-9.

6 Bernell O, Lapidus A, Hellers G. Risk factors for surgery and postoperative recurrence in Crohn's disease. Ann Surg 2000;231:38-45.

7 Sprakes MB, Ford AC, Suares NC, et al. Costs of care for Crohn's disease following the introduction of infliximab: a single-centre UK experience. Aliment Pharmacol Ther 2010;32:1357-63.

8 Loomes DE, Teshima C, Jacobs P, et al. Health care resource use and costs for Crohn's disease before and after infliximab therapy. Can J Gastroenterol 2011;25:497-502.

9 NHS Reference Costs. Department of Health. England. 2010-2011.

10 Joint Formulary Committee. British national formulary. 66th edn. London: BMJ Group and Pharmaceutical Press, 2013.
11 Panés J, Guilera M, Ginard D, et al. Treatment cost of ulcerative colitis is apheresis with Adacolumn cost-effective? Dig Liver Dis 2007;39:617-25.

12 NICE Technology Appraisal Guidance 187. Inflximab (review) and adalimumab for the treatment of Crohn's disease. http:// www.nice.org.uk/nicemedia/live/12985/48552/48552.pdf.

13 Mowat C, Cole A, Windsor A, et al. Guidelines for the management of Inflammatory Bowel Disease in adults. Gut 2011;60:571-607.

14 Eaden JA, Abrams KR, Mayberry JF. The risk of colorectal cancer in ulcerative colitis: a metaanalysis. Gut 2001;48:526-35.

15 NICE costing statement: Colonic surveillance for prevention of colorectal cancer in patients with ulcerative colitis, Crohn's disease or adenomas. 2011.

16 Wolff B, Fleshman JW, Beck DE, et al. The ASCRS textbook of colon and rectal surgery. New York: Springer, 2007.

17 Bernstein CN, Blanchard JF, Rawsthorne P, et al. The prevalence of extraintestinal diseases in inflammatory bowel disease: a population-based study. Am J Gastroenterol 2001;96:1116-22.

18 Gleason PP, Alexander GC, Starner CI, et al. Health plan utilization and costs of specialty drugs within 4 chronic conditions. J Manag Care Pharm 2013;19:542-8.

19 Bassi A, Dodd S, Williamson P, et al. Cost of illness of inflammatory bowel disease in the UK: a single centre retrospective study. Gut 2004;53:1471-8. 